\title{
Possible contamination of organ preservation fluid with Bacillus cereus: the United Kingdom response
}

\author{
C S Brown (colin.brown@hpa.org.uk), 1,2, M A Chand ${ }^{1,3}$, P Hoffman 4 , N Woodford5, D M Livermore 5 , S Brailsford 6 , S Gharbia7, N \\ Small $^{8}$, E Billingham 9 , M Zambon ${ }^{1}, K$ Grant $^{10}$, on behalf of the United Kingdom incident response team \\ 1. Microbiology Services Division, Health Protection Agency, London, United Kingdom \\ 2. Centre for Clinical Infection and Diagnostics Research, King's College London, London, England \\ 3. University College London Hospitals, London, United Kingdom \\ 4. Laboratory of Healthcare-associated Infection, Health Protection Agency, London, United Kingdom \\ 5. Antibiotic Resistance Monitoring \& Reference Laboratory, Health Protection Agency, London, United Kingdom \\ 6. Immunisation, Hepatitis and Blood Safety Department, Health Protection Agency, London, United Kingdom \\ 7. Department of Bioanalysis and Horizon Technologies - Applied and Functional Genomics Unit, Health Protection Agency, \\ London, United Kingdom \\ 8. Imaging Acute and Community Care, Medicines and Healthcare products Regulatory Agency, London, United Kingdom \\ 9. NHS Blood and Transplant, London, United Kingdom \\ 10. Laboratory of Gastrointestinal Pathogens, Health Protection Agency, London, United Kingdom
}

Brown CS, Chand MA, Hoffman P, Woodford N, Livermore DM, Brailsford S, Gharbia S, Small N, Billingham E, Zambon M, Grant K, on behalf of the United Kingdom incident response team. Possible contamination of organ preservation fluid with Bacillus cereus: the United Kingdom response. Euro Surveill. 2012;17(18):pii=20165. Available online: http://www.eurosurveillance.org/ViewArticle.aspx?Articleld=20165

We describe here the United Kingdom (UK) response following the recent international recall of an organ preservation fluid owing to potential Bacillus cereus contamination. This fluid is used for the transport of solid organs and pancreatic islet cells for transplant. We detail the response mechanisms, including the initial risk stratification, investigatory approaches, isolate analysis and communications to professional bodies. This report further lays out the potential need for enhanced surveillance in UK transplant patients.

\section{Current incident}

On 23 March 2012, Bristol-Meyers Squibb notified the Medicines and Healthcare products Regulatory Agency - an executive agency of the Department of Health, England - of possible contamination of their product ViaSpan, an organ preservation fluid used for the transport of solid organs (liver, kidney, bowel and pancreas) and pancreatic islet cells for transplant [1]. Bacillus cereus contamination from the production line was identified on 16 March 2012 through a simulated production run in February 2012 that used bacterial growth medium instead of ViaSpan [2], designed to be a worst-case challenge to the microbiological integrity of the production process [3]. The contaminant load is unknown. This routine production simulation run had last been performed in July 2011, with satisfactory results. To date, there has been no evidence of contamination in batches of ViaSpan produced before or since contamination was found in the simulated production run in February 2012. Nevertheless, a precautionary international recall of ViaSpan was issued to relevant regulatory authorities on 29 March 2012 and to product end-users on 30 March 2012 [4]. Investigations by the manufacturer concluded that the most probable cause was a manufacturing failure [5].

\section{Background}

$B$. cereus is a well-known cause of food poisoning; however, it can also cause serious invasive disease including bacteraemia, septicaemia, endocarditis, osteomyelitis, pneumonia, brain abscess, and meningitis in severely immunocompromised patients, such as those with haematological malignancy, and in patients with indwelling vascular catheters [6]. Previous contamination of medical fluids [7] and devices [8] with $B$. cereus has been reported.

\section{United Kingdom response}

A coordinated response involving the Medicines and Healthcare products Regulatory Agency (MHRA), NHS Blood and Transplant (NHSBT) and the Health Protection Agency (HPA) was undertaken to quantify the potential risks to patients; the Department of Health and other United Kingdom (UK) devolved nations' health administrations were also involved. The different organisations liaised via regular teleconferences, meetings and email, ensuring all information was readily available in adequate time to be sent out to the transplant community by way of a daily email. A risk assessment was conducted for patients already transplanted with organs transported in potentially contaminated fluid and for those who could potentially be affected by the remaining ViaSpan stock. The continued use of implicated batches of ViaSpan was weighed against the risk of deferred transplantation resulting from the lack of an immediately available licensed alternative. Despite the potential contamination of ViaSpan with $B$. cereus and given the scarcity of donor organs and high mortality of patients on waiting lists for solid organ transplants, it was deemed that patients were at a much greater risk through not receiving a transplant than by the continued use of a potentially contaminated product. 
Advice was issued to clinicians with responsibilities for transplant patients about alternative fluids. Where no suitable alternative was available, the manufacturer's advice that the solution could be used with caution was supported, together with advice to send a sample of fluid from any implicated batch of Viaspan for culture, to inform the surgical and renal teams of the results, to remain vigilant for signs of infection or transplant rejection, and to consider modifying prophylactic or therapeutic antimicrobial administration to cover $B$. cereus infection [9]. $B$. cereus produces multiple betalactamases and is commonly, though variably, resistant to penicillins, including beta-lactamase inhibitor combinations, carbapenems and cephalosporins.

\section{Surveillance data}

Routine laboratory data on reported cases of either $B$. cereus or all Bacillus species blood culture isolates in the UK showed no increase in systemic infections since July 2011 (Table 1). There were 31 reported isolates of $B$. cereus from blood cultures between July 2011 and March 2012 compared with a mean of 40 over comparable nine-month periods in the previous four years. The proportion of $B$. cereus isolates from blood culture $(22.6 \%)$ was very similar to the mean for the previous four years $(24.0 \%)$. No changes in the number of reports of $B$. cereus isolates in the HPA LabBase surveillance reporting system from 2007 to 2012 were seen (Figure, displayed with quarterly moving averages).

Of the small numbers of clinical $B$. cereus isolates with recorded clinical information that were sent to HPA reference laboratories for further identification $(n=24)$, none was reported as being from a transplant patient (Table 2). A large proportion of isolates were from patients with probable haematological or other malignancy. These are highly immunosuppressed patients and it is likely that referral of these samples reflects the fact that clinicians appropriately recognise $B$. cereus as a possible pathogen with the potential for serious morbidity or mortality rather than a sporadic contaminant in this context. The same approach should be applied to solid organ transplant patients.

As invasive infection with opportunistic Bacillus species (apart from B. anthracis) is not subject to mandatory notification in the UK, transplant centres were also requested to determine from local laboratories whether there had been any $B$. cereus infections in patients since mid-2011. NHS Blood and Transplant also reviewed similar information within their clinical reporting system and did not note any increase in adverse events since July 2011. It is plausible, though unlikely, that transmission of $B$. cereus may be missed because transplant recipients are given appropriate prophylactic antimicrobials.

Databases in solid organ transplant centres were interrogated for possible linkages with laboratory reports of isolation of $B$. cereus. Of five centres that routinely culture fluids, only one reported detection of Bacillus species from July 2011 onwards. This was a lower frequency than that for the preceding six months, and Bacillus species were isolated only from enrichment cultures (with additional growth factors) at $25{ }^{\circ} \mathrm{C}$, as opposed to standard blood culture incubation at $37{ }^{\circ} \mathrm{C}$ (Table 3 ). Thus there is currently no evidence from any existing surveillance system of any increase in $B$. cereus bacteraemias or of any other infections in transplant patients since July 2011.

\section{Bacillus cereus isolates}

Six isolates from the bacterial growth medium were forwarded in duplicate by the manufacturer to the HPA for confirmation, typing and antimicrobial susceptibility testing to ensure that appropriate advice was available to healthcare providers. The selection method for

TABLE 1

Bacillus cereus and Bacillus species blood culture and other clinical isolates captured by the Health Protection Agency LabBase surveillance reporting systemª, United Kingdom, each July to March 2007-2012 (n=3,043)

\begin{tabular}{|c|c|c|c|c|c|c|c|}
\hline \multirow[b]{2}{*}{ Reporting period } & \multicolumn{3}{|c|}{ Isolates of Bacillus cereus } & \multicolumn{3}{|c|}{ Isolates of Bacillus species } & \multirow[b]{2}{*}{ Overall } \\
\hline & $\begin{array}{c}\text { Number from } \\
\text { blood culture } \\
\text { (\%) }\end{array}$ & $\begin{array}{l}\text { Number from } \\
\text { other clinical } \\
\text { sites }\end{array}$ & Total & $\begin{array}{c}\text { Number from } \\
\text { blood culture } \\
\text { (\%) }\end{array}$ & $\begin{array}{l}\text { Number from } \\
\text { other clinical } \\
\text { sites }\end{array}$ & Total & \\
\hline Jul 2007-Mar 2008 & $46(22.7)$ & 157 & 203 & $338(69.4)$ & 149 & 487 & 690 \\
\hline Jul 2008-Mar 2009 & $36(23.7)$ & 116 & 152 & $236(70.0)$ & 101 & 337 & 489 \\
\hline Jul 2009-Mar 2010 & $42(24 \cdot 4)$ & 130 & 172 & $242(56.5)$ & 186 & 428 & 600 \\
\hline Jul 2010-Mar 2011 & $37(26.2)$ & 104 & 141 & $223(42.8)$ & 298 & 521 & 662 \\
\hline Mean Jul 2007-Mar 2011 & $40(24.0)$ & 127 & 167 & $260(58.7)$ & 184 & 443 & 610 \\
\hline Jul 2011-Mar 2012 & $31(22.6)$ & 106 & 137 & $195(41.9)$ & 270 & 465 & 602 \\
\hline Total & $192(23.9)$ & 613 & 805 & $1,234(55.1)$ & 1,004 & 2,238 & 3,043 \\
\hline
\end{tabular}

a LabBase obtains data from all National Health Service laboratories by an automated data extract with manual final approval. It records only positive results for selected organisms $(n=2,500)$ and is used to generate exceedance scores [10]. 
these isolates was unclear. The isolates were confirmed as $B$. cereus by a combination of $16 \mathrm{~S}$ and gyrase $B$ gene sequencing and phenotypic tests, which included confirming the absence of parasporal crystals [11]. The six isolates were subtyped by fluorescent amplified fragment length polymorphism (fAFLP) analysis and two very similar profiles were obtained, indicating that all isolates belonged to one of two closely related genetic groups (data not shown). The minor band differences may be due to single nucleotide polymorphism(s), however, and the two fAFLP types may actually represent the same strain.

In vitro studies using Etests on Iso-Sensitest agar [12] showed that the isolates were resistant to penicillins and extended-spectrum cephalosporins, reflecting beta-lactamase production. Despite high activity of meropenem in vitro (minimum inhibitory concentrations (MICs) $\leq 0.064 \mathrm{mg} / \mathrm{L}$ ), concerns remain over inducible resistance since Bcll - a chromosomal metallo-betalactamase that is widespread in $B$. cereus - has carbapenemase activity [13-15]. Where possible, the European Committee on Antimicrobial Susceptibility Testing (EUCAST) guidelines [16] were followed in interpreting MICs; however, there are no specific breakpoints for $B$. cereus.

The isolates were not susceptible to vancomycin (MICs $4 \mathrm{mg} / \mathrm{L}$ ) and, unusually, also were resistant to daptomycin (MICs 2-4 mg/L), suggesting differences in membrane composition compared with other collections of Bacillus species reported to be susceptible (MIC $_{50}$ and
$\mathrm{MIC}_{90}$ values of 0.25 and $1 \mathrm{mg} / \mathrm{L}$, respectively) to this lipopeptide [17].

\section{Risk management}

A bactericidal agent would be preferred to a bacteriostatic agent in immunosuppressed patients. The six isolates from the bacterial growth medium were susceptible to the following antibacterical agents: ciprofloxacin (MICs $\leq 0.25 \mathrm{mg} / \mathrm{L}$ ), gentamicin (MICs $\leq 0.5$ $\mathrm{mg} / \mathrm{L}$ ), and, with the earlier caveat, meropenem. They also were susceptible to tetracyclines (rank order of MICs: tetracycline, $\leq 0.25 \mathrm{mg} / \mathrm{L}$; doxycycline, $\leq 0.125$ $\mathrm{mg} / \mathrm{L}$; tigecycline, $\leq 0.06 \mathrm{mg} / \mathrm{L}$ ) and to linezolid (MICs $\leq 0.5 \mathrm{mg} / \mathrm{L}$ ), which are all bacteriostatic. These susceptibilities were included in a detailed rapid risk assessment produced by the European Centre for Disease Prevention and Control (ECDC), to ensure a harmonised European approach to procurement of alternative supplies, surveillance and clinical management [18].

Bacteriological culture of the implicated Viaspan batches is recommended for each transplant, with any positive cultures being reported to NHS Blood and Transplant [19]. Ongoing consultation with the manufacturer will investigate the root cause of $B$. cereus ingress into the production line, which will inform risk assessment, alongside further validation of the integrity of the production process.

The manufacturer notified all countries in the EU and European Economic Area that used the product, and a rapid alert notification was issued by the Austrian Medicines Authority on 29 March 2012 to further

\section{FIGURE}

Cases of Bacillus cereus infection (isolates from blood culture ( $\mathrm{n}=261)$ and other clinical sites $(\mathrm{n}=855)$ ) captured by the Health Protection Agency LabBase surveillance reporting systema, United Kingdom, January 2007-March 2012

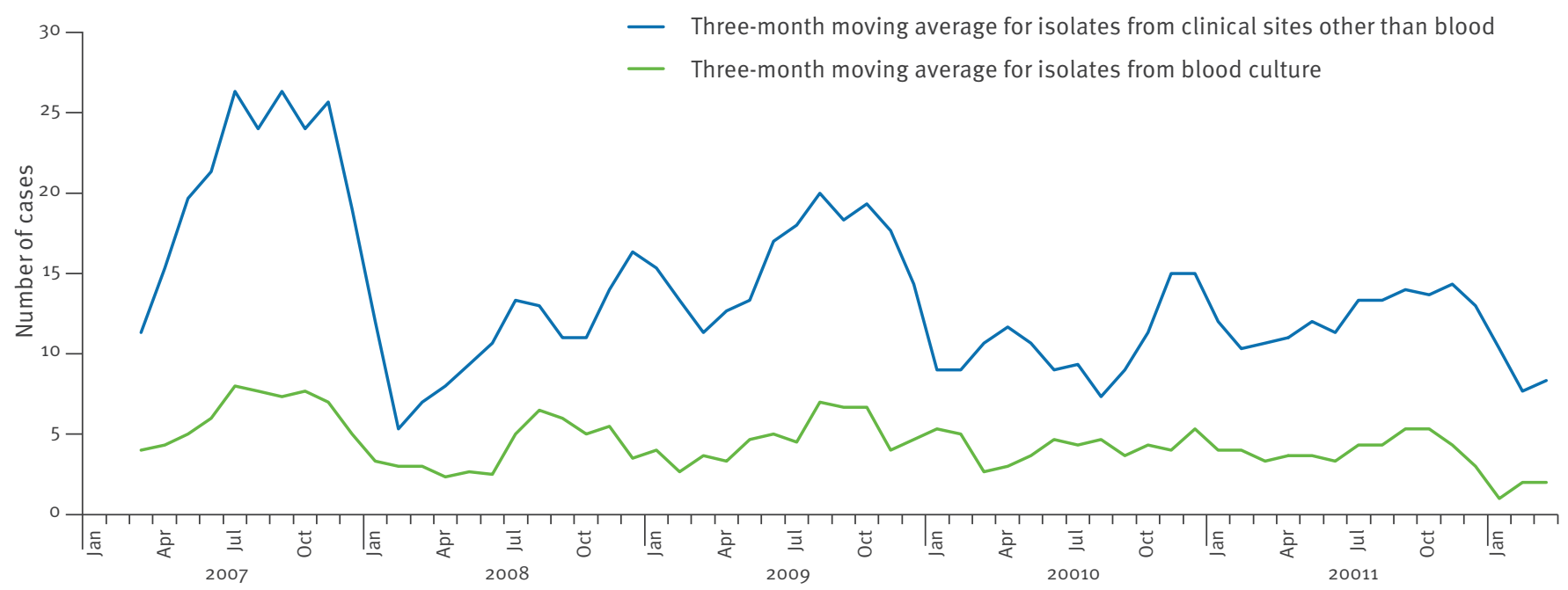

LabBase obtains data from all National Health Service laboratories by an automated data extract with manual final approval. It records only positive results for selected organisms $(n=2,500)$ and is used to generate exceedance scores [10]. 


\section{TABLE 2}

Underlying conditions in 24 patients with Bacillus cereus blood culture isolates referred to the Health Protection Agency Colindale ${ }^{\text {a }}$, United Kingdom, each July to March 2010-2012

\begin{tabular}{|l|c|}
\hline Underlying condition & \multicolumn{1}{|c|}{$\begin{array}{c}\text { Number of patients with } \\
\text { B. cereus blood culture isolates }\end{array}$} \\
\hline $\begin{array}{l}\text { Probable haematological } \\
\text { malignancy }\end{array}$ & 10 \\
\hline Oncological malignancy & 3 \\
\hline $\begin{array}{l}\text { Long-term intravenous catheter in } \\
\text { situ (with or without malignancy) }\end{array}$ & 1 \\
\hline Endocarditis & 1 \\
\hline Intravenous drug use & 5 \\
\hline $\begin{array}{l}\text { No underlying risk factors - } \\
\text { patients had non-defined sepsis }\end{array}$ & 24 \\
\hline Total & \multicolumn{2}{|c|}{} \\
\hline
\end{tabular}

a These isolates are referred by microbiologists for confirmation and antimicrobial susceptibility testing, the criteria for referral being based on clinician interest or concern. Thus, they represent a subset of total LabBase isolates. LabBase obtains data from all National Health Service laboratories by an automated data extract with manual final approval. It records only positive results for selected organisms $(n=2,500)$ and is used to generate exceedance scores [10].

advise EU Member States of the recall of the product [20]; at present we have no further information on the response of other countries. The proposed action was to recall implicated fluids if alternative products were available. If no alternative product was available, the manufacturer would contact the country to discuss maintenance of the existing supply. The Medicines and Healthcare products Regulatory Agency also notified all Member States' medical device regulatory authorities about the recall.

Supply chains were also managed to ensure that suitable alternatives were sourced, and perfusion protocols amended to reflect the change in transplant transport fluid.

\section{Conclusions}

This incident underscores the need for robust structured surveillance of solid organ transplant patients, to include reporting of adverse incidents and infections, as acknowledged by the recent EU directive [21]. This sets common standards for organ donation and transplantation across Europe, including mandatory reporting and management systems for serious adverse events. The outcome and survival of patients following organ transplants is monitored in the UK by NHS Blood and Transplant and reported by a dedicated statistics unit, with serious adverse events following transplantation reported to their Organ Donation and Transplantation Directorate (ODT) clinical governance system. This is a passive surveillance system relying on voluntary reporting, in addition to a clinical monitoring

\section{TABLE 3}

Bacillus species isolated, data from reporting transplant centres $^{\mathrm{a}}$ that routinely culture organ transplant fluid post-organ transfer for transplantation, United Kingdom, February 2011-July 2011 and July 2011-March 2012 (n=7)

\begin{tabular}{|l|l|l|}
\multirow{2}{*}{ Centre } & \multicolumn{2}{|l|}{ Number of fluids with Bacillus species isolated } \\
\cline { 2 - 3 } & Feb 2011-Jul 2011 & Jul 2011-Mar 2012 \\
\hline A & Not assessed & 0 \\
\hline B & Not assessed & 0 \\
\hline C & Not assessed & 0 \\
\hline D & Not assessed & 0 \\
\hline E & $4^{\text {b }}$ & 3 \\
\hline
\end{tabular}

Transplant centres that report to the NHS Blood and Transplant. b Isolated only from enrichment cultures grown at $25^{\circ} \mathrm{C}$ (according to the laboratory's standard operating procedure).

system where clinicians are encouraged to report poor outcomes of transplantation or other issues of concern. There is currently no routine surveillance system for infections in donors or recipients post-transplant, and only events deemed as serious adverse events are reported routinely. Historically, it has been difficult to establish infection surveillance systems for organ transplants. Unlike for blood and tissue donation, infection surveillance testing of donors and recipients is carried out at many different centres across the UK. The introduction of an electronic systemic would facilitate surveillance post-organ transplantation and facilitate rapid risk assessments. In addition, NHS Blood and Transplant have agreed that not only the fluid type used but also the batch number will be recorded in future, in light of this incident.

This product recall serves as a general reminder that specialist sectors of healthcare that have both vulnerable patients and unusual infections may need to be able to establish rapidly new or enhanced surveillance systems in response to real or potential emerging infections.

\section{Acknowledgements}

This article was written with the assistance and on behalf of the UK incident response team, comprising the Department of Health (England) and devolved nations' health administrations, Medicines and Healthcare products Regulatory Agency, NHS Blood and Transplant and Health Protection Agency. The authors would like to thank Bob Adak and the Department of Gastrointestinal, Emerging and Zoonotic Infections for their help with collection and analysis of the data; Robert Hill, Unit Head, Antibiotic Resistance Monitoring and Reference Laboratory for his invaluable contribution to the initial advice and comments on this paper; and to Nicola 
Lennard, Deputy Clinical Director, Medicines and Healthcare products Regulatory Agency. In addition, many thanks to the reporting centres for NHS Blood and Transplant data analysis - Oxford, Scotland, Leeds, Nottingham and Cambridge.

\section{References}

1. Southard JH, Belzer FO. Organ preservation. Annu Rev Med. 1995:46;235-47.

2. Medicines and Healthcare products Regulatory Agency (MHRA). Precautionary safety advice to transplant centres about Viaspan organ preservation solution manufactured by Bristol Myers Squibb Pharmaceuticals. London: MHRA. [Accessed 25 Apr 2012]. Available from:

http://www.mhra.gov.uk/Safetyinformation/

Healthcareproviders/Precautionarysafetyadvicetotransplantce ntresaboutViaspanorganpreservationsolutionmanufacturedby BristolMyersSquibbPharmaceuticals/index.htm

3. European Commission (EC). Manufacture of sterile medicinal products (corrected version). Annex 1. In: EU guidelines to Good Manufacturing Practice. Medicinal products for human and veterinary use. Volume 4. Brussels; EC; 2008. Available from: http://ec.europa.eu/health/files/eudralex/ vol-4/2008_11_25_gmp-an1_en.pdf

4. Bristol-Meyers Squibb (BMS). Company statement on VIASPAN ${ }^{\circledR}$. Rueil-Malmaison: BMS. Press release. [Accessed 25 April 2012]. Available from: http://www.bms.com/news/ features/2012/Pages/viaspan-03302012.aspx

5. Bristol-Myers Squibb (BMS). Bristol-Myers Squibb final quality risk assessment. Rueil-Malmaison: BMS; 11 April 2012.

6. Bottone EJ. Bacillus cereus, a volatile human pathogen. Clin Microbiol Rev. 2010;23(2):382-98.

7. Thuler LC, Velasco E, de Souza Martins CA, de Faria LM, da Fonseca NP, Dias LM, et al. An outbreak of Bacillus species in a cancer hospital. Infect Control Hosp Epidemiol. 1998:19(11):856-8.

8. Van Der Zwet WC, Parlevliet GA, Savelkoul PH, Stoof J, Kaiser AM, Van Furth AM, et al. Outbreak of Bacillus cereus infections in a neonatal intensive care unit traced to balloons used in manual ventilation. J Clin Microbiol. 2000;38(11):4131-6.

9. Medicines and Healthcare products Regulatory Agency (MHRA), Health Protection Agency (HPA) and NHS Blood \& Transplant (NHSBT). Possible contamination of Viaspan organ transport fluid. Information for clinicians. MHRA, HPA and NHSBT; 5 April 2012. Available from: http://www.mhra.gov.uk/home/groups/ comms-ic/documents/websiteresources/con149657.pdf

10. Health Protection Agency (HPA). Major changes to reporting practices for laboratory communicable disease reporting. London: HPA. [Accessed 6 Apr 2012]. Available from: http:// www.hpa.org.uk/ProductsServices/InfectiousDiseases/ ServicesActivities/Surveillance/SourcesOfSurveillanceData/ survGuidanceChanges/

11. Vilas-Bôas GT, Peruca AP, Arantes OM. Biology and taxonomy of Bacillus cereus, Bacillus anthracis, and Bacillus thuringiensis. Can J Microbiol. 2007;53:673-87

12. Andrews JM, Wise R. Susceptibility testing of Bacillus species. J Antimicrob Chemother. 2002;49(6):1040-2.

13. Kiyomizu K, Yagi T, Yoshida H, Minami R, Tanimura A, Karasuno $T$, et al. Fulminant septicemia of Bacillus cereus resistant to carbapenem in a patient with biphenotypic acute leukemia. J Infect Chemother. 2008;14(5):361-7.

14. Katsuya H, Takata T, Ishikawa T, Sasaki H, Ishitsuka K, Takamatsu Y, et al. A patient with acute myeloid leukemia who developed fatal pneumonia caused by carbapenem-resistant Bacillus cereus. J Infect Chemother 2009;15(1):39-41.

15. Hussain M, Carlino A, Madonna MJ, Lampen JO. Cloning and sequencing of the metallothioprotein beta-lactamase Il gene of Bacillus cereus $569 / \mathrm{H}$ in Escherichia coli. J Bacteriol. 1985;164(1):223-9.

16. European Committee on Antimicrobial Susceptibility Testing (EUCAST). Clinical breakpoints. EUCAST. [Accessed 6 Apr 2012]. Available from: http://www.eucast.org/ clinical_breakpoints/

17. Luna VA, King DS, Gulledge J, Cannons AC, Amuso PT, Cattani J. Susceptibility of Bacillus anthracis, Bacillus cereus, Bacillus mycoides, Bacillus pseudomycoides and Bacillus thuringiensis to 24 antimicrobials using Sensititre automated microbroth dilution and Etest agar gradient diffusion methods. J Antimicrob Chemother. 2007;60(3):555-67.
18. European Centre for Disease Prevention and Control (ECDC). Potential contamination of Viaspan ${ }^{\circledR}$ organ perfusion solution. Stockholm: ECDC; 23 April 2012. Rapid risk assessment. Available from: http://www.ecdc.europa.eu/en/publications/ Publications/1204-TER-Risk-assessment-contaminationperfusion-solution.pdf

19. Advisory Committee on the Safety of Blood, Tissues and Organs (SaBTO). Guidance on the microbiological safety of human organs, tissues and cells used in transplantation. London: SaBTO; February 2011. Available from: http://www. dh.gov.uk/prod_consum_dh/groups/dh_digitalassets/ documents/digitalasset/dh_130515.pdf

20. Austrian Medicines Authority. Rapid alert notification AT/ II/23/01. Vienna: Austrian Medicines Authority; 29 March 2012 Confidential report.

21. European Commission. Directive 2010/45/EU of the European Parliament and of the Council of 7 July 2010 on standards of quality and safety of human organs intended for transplantation. Luxembourg: Publications Office of the European Union. 6.8.2010:L 207/14. Available from: http://eurlex.europa.eu/LexUriServ/LexUriServ.do?uri=0J:L:2010:207:00 14:0029:EN:PDF 